Lin Gao, Jiang Zhao, Guo-zheng Quan*, Wei Xiong* and Chao An

\title{
Study on the Evolution of Damage Degradation at Different Temperatures and Strain Rates for Ti-6Al-4V Alloy
}

https://doi.org/10.1515/htmp-2018-0091

Received June 03, 2018; accepted August 14, 2018

\section{Introduction}

Titanium alloys have been extensively used in aircraft fabrication, ship building, energy and chemical industries due to their attractive strength-to-weight, good fatigue properties, high toughness and great corrosion resistance $[1,2]$. Additionally, they are also applied in biomedicine such as artificial joints and dental implants and so on due to their excellent biocompatibility [3, 4]. Ti-6Al-4V alloy, as a typical $\alpha+\beta$ titanium alloy, is earlier developed and studied, and now accounts for the largest share of the market. Currently, the components of Ti-6Al-4V alloys are usually manufactured by hot plastic processing with low cost and short cycle. Nevertheless, hot plastic forming processes are always accompanied by notable damage accumulation causing internal deterioration which may lead to the occurrence of macroscopic failure. The most common macroscopic failure during such process could be fracture caused ductile damage. Thus, it is an important issue to carry out the desired products without any fracture in the plastic forming processes. However, the empirical know-how of the worker is crucial for the fracture-free products in industrial practice at present, which usually requires very costly trial-and-error [5]. Therefore, it is significant to establish an effective theoretical method to predict the initial ductile fracture time, and then avoid the fracture by choosing appropriate technological parameters, which could obviously save time and costs [6,7].

Over the last few decades, considerable efforts have been made to investigate the ductile fracture during hot plastic deformation. Cockcroft-Latham fracture criteria has been demonstrated to be a good way for evaluating ductile fracture value in hot plastic forming simulation and applied successfully to a variety of loading situations [6-10]. However, Cockcroft and Latham considered the critical damage values as a constant of materials like yield stress. But in fact the critical damage values can be described as a function of local temperature, strain, and the stress state [11]. It was known that the resulting ductile fracture value during plastic processes can be calculated with the model of effective stresses, considering crack closure effects by splitting the Cauchy stress tensor in a 
compressive and tensile part [12]. Thus the materials damage during forming processes can be simulated by implementing damage models into finite element model. The ductile fracture tendency can be characterized as the ration of damage value, namely ductile fracture criteria (DFC) $[13,14]$. Tvergaard and Needleman [11] demonstrated the dramatic influence of temperature on the critical damage value in a ductile fracture process and applied it into finite element analysis. Further, Quan et al. [12] pointed out that temperature and strain rate markedly influenced the critical damage value, and put forward an innovative concept of damage sensitive rate as the essential intermediate quantity, and then obtained the varying ductile fracture criteria (VDFC) of 3Cr20Ni10W2 austenitic heat-resistant alloy. Several such works analyze the ductile fracture behaviors of alloys and established the DFC, but few researches on the initial ductile fracture time during hot deformation along with processing parameters.

The main purpose of this paper is to analyze effects of temperature and strain rate on ductile damage evolution for Ti-6Al-4V alloy, and develop DFC histogram along with deforming parameters (temperature and strain rate), and then identify the initial ductile fracture time. In the present work, a series of compressions of Ti-6Al-4V alloy with a height reduction range of $60 \%$ were performed in the temperature range of 1023-1323 $\mathrm{K}$ and the strain rate range of $0.01-10 \mathrm{~s}^{-1}$ on a Gleeble-3500 thermo-mechanical simulator. An approach to identify the initial fracture time was carried out and an innovative concept of damage sensitive rate was used as the essential intermediate quantity. Then based on the stress-strain data achieved from the compression tests, the FE model was built and the FE analysis under seven temperatures and four strain rate were conducted, and then the critical damage values at such conditions were obtained. Subsequently, the varying ductile fracture criteria (VDFC) was established, and then the initial fracture time of different temperatures and different strain rate were identified based on the VDFC. In addition, to verify the VDFC, several typical microstructures of Ti-6V-4Al alloy with a high risk of fracture were observed by optical microscopy and compared with the CDFC.

\section{Hot compression test}

\section{Experimental procedures}

The chemical compositions of Ti-6Al-4V alloy used in this work are as follows (wt.\%): $6.02 \mathrm{Al}, 3.91 \mathrm{~V}, 0.13 \mathrm{O}$,
$0.08 \mathrm{Fe}, 0.008 \mathrm{~N}$, balance Ti. The beta-transus temperature of such alloy is approximately $1263 \mathrm{~K}$. The following experimental procedures follow ASTM Standard: E209-00. An extruded bar with a diameter of $200 \mathrm{~mm}$ was as-received material. Twenty-eight cylindrical specimens with $10 \mathrm{~mm}$ in diameter and $12 \mathrm{~mm}$ in height were machined from the bar by wireelectrode cutting. Hot compression tests were carried out in the temperature range of 1023-1323 K with an interval of $50 \mathrm{~K}$ and true strain rates of $0.01 \mathrm{~s}^{-1}$, $0.1 \mathrm{~s}^{-1}, 1 \mathrm{~s}^{-1}$ and $10 \mathrm{~s}^{-1}$ on a Gleeble 3500 (GSI SLV $\mathrm{GmbH}$ Co. Germany) thermal mechanical simulator with a fully integrated digital closed loop control thermal and mechanical testing system produced by DSI. During a compressing process, the specimen was resistance heated to the designed temperature at a heating rate of $10 \mathrm{~K} / \mathrm{s}$ and held at that temperature for $180 \mathrm{~s}$ by thermo-coupled-feedback-controlled AC current, aiming to obtain a homogeneous heat distribution and decrease the material anisotropy. Then the specimen was compressed to a true strain 0.916 (height reduction of $60 \%$ ) under the designed temperature and strain rate. After the compression, the deformed specimen was water-quenched instantly to insure a uniform temperature field to retain the organizational form at elevated temperature. Twenty-eight compression tests under seven temperatures and four strain rates were performed according to such procedure. It is worth pointing out that in this work only one sample was used in a certain loading condition. This is due to the fact that Gleeble 3500 thermal mechanical simulator controls temperature raise, temperature holding and compressing speed loading precisely by a fully integrated digital closed loop control thermal and mechanical testing system. It is certain that all the collected true stress-strain data accord with other's work.

During these compressing processes, the variations of stress and strain were monitored continuously by a personal computer equipped with an automatic data acquisition system. The true stress-strain relationships were derived from the measurement of the nominal stress-strain curves collected according to the following formula: $\sigma_{\mathrm{T}}=\sigma_{\mathrm{N}}\left(1+\varepsilon_{\mathrm{N}}\right), \varepsilon_{\mathrm{T}}=\ln \left(1+\varepsilon_{\mathrm{N}}\right)$, where $\sigma_{\mathrm{T}}$ is the true stress, $\sigma_{\mathrm{N}}$ is the nominal stress, $\varepsilon_{\mathrm{T}}$ is the true strain and $\varepsilon_{\mathrm{N}}$ is the nominal strain [15].

\section{Flow stress behaviors}

Figure 1 shows the stress-strain data collected from the compression tests at different seven temperatures and 
four strain rates. Comparing these curves with one another, it is summarized that the flow behaviors show a noticeable dependence on deformation conditions including temperature, strain and strain rate. Generally, the stress level decreases with increasing temperature at a certain strain rate and decreasing strain rate at a certain temperature. This is due to the fact that for the strain-softening alloy as Ti-6Al-4V, lower strain rate and higher temperature provides enough time and energy for the dislocation accumulation and atom diffusion. It is widely accepted that the evolution of flow stress indicates the intrinsic interactions between work hardening (WH) and softening mechanisms during hot deformation. There are two different types of softening mechanism along with the studied temperature range, i.e. DRV (dynamic recovery) in $\beta$-phase and DRV + DRX (dynamic recrystallization) in $\alpha+\beta$-phase. In $\beta$-phase temperatures (1273 $\mathrm{K}$ and $1323 \mathrm{~K}$ ), flow stress sharply increases to a certain value, and then keeps a relatively steady state due to the balance between $\mathrm{WH}$ and DRV. This kind of flow stress evolution attributes to DRV softening type. The presented temperature-rate dependent behaviors of Ti$6 \mathrm{Al}-4 \mathrm{~V}$ alloy in $\beta$-phase temperatures agree with the data in the reference [3]. In $\alpha+\beta$-phase temperatures $(1023 \mathrm{~K}, 1073 \mathrm{~K}, 1123 \mathrm{~K}, 1173 \mathrm{~K}$ and $1223 \mathrm{~K})$, flow stress rapidly increases before the strain of 0.2 , which is caused by severe work hardening (WH). Following which, flow stress increases slower and slower till a single peak value due to the onset and enhancement of $\mathrm{DRV}+\mathrm{DRX}$ of $\alpha$-phase, subsequently, flow stress decreases gradually to a steady value due to a balance between reinforce induced by $\mathrm{WH}$ and softening deduced by DRV + DRX. This kind of flow stress evolution attributes to DRX softening type. The presented temperature-rate dependent behaviors of Ti$6 \mathrm{Al}-4 \mathrm{~V}$ alloy in $\alpha+\beta$-phase temperatures agree with the data in the reference [2-4]. Beside, these stressstrain data are the important foundation of calculating damage in following sections.

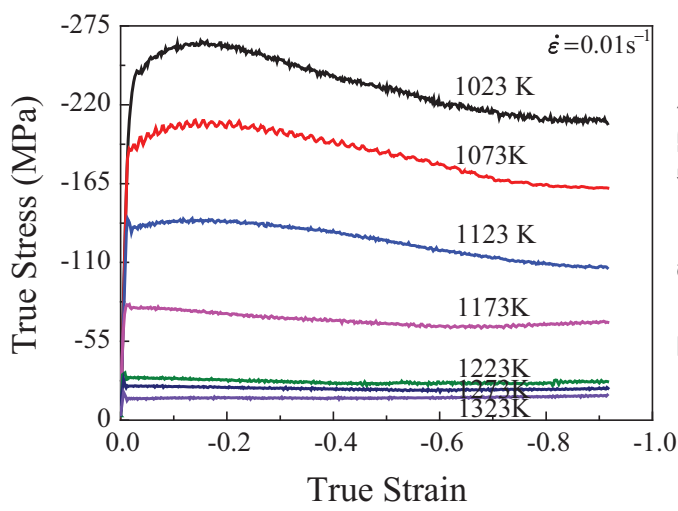

(a)

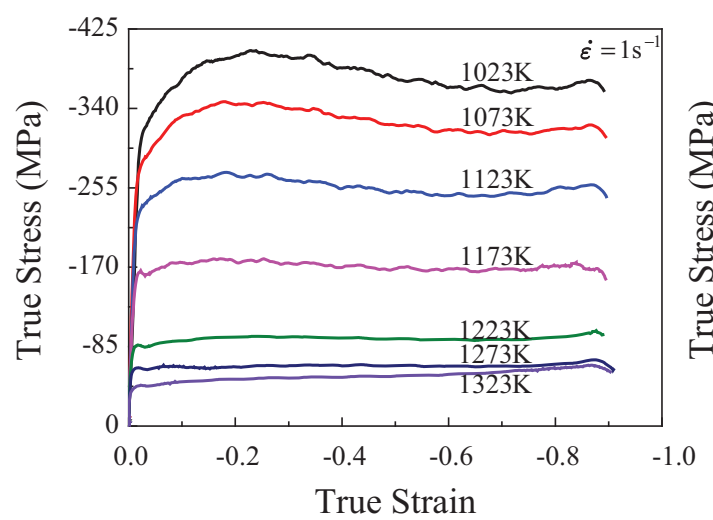

(c)

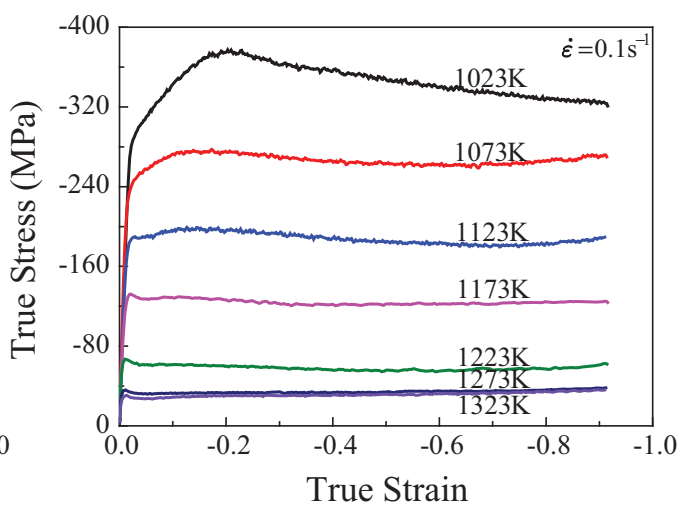

(b)

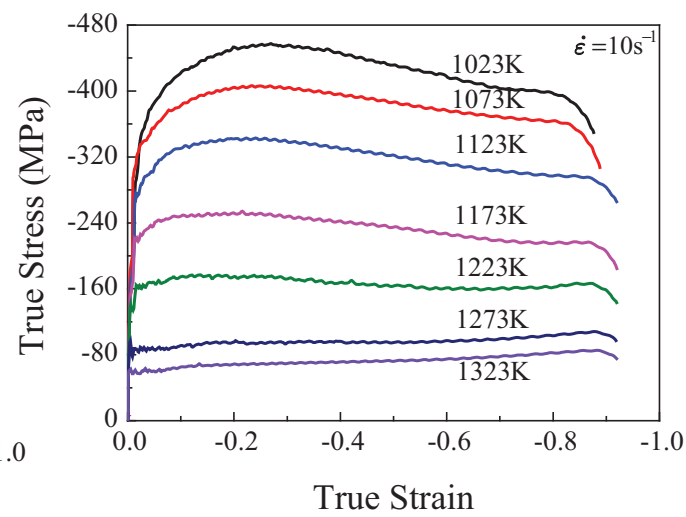

(d)

Figure 1: The true stress-strain curves of Ti-6Al-4V alloy under the different deformation temperatures with strain rates (a) $0.01 \mathrm{~s}^{-1}$, (b) $0.1 \mathrm{~s}^{-1}$, (c) $1 \mathrm{~s}^{-1}$, (d) $10 \mathrm{~s}^{-1}$. 


\section{Basis for damage computation}

\section{Cockcroft-Latham's DFC}

The workability of metal materials plays a major role for judging whether the metal will be manufactured successfully or caused ductile fracture in the forming processes. In general, the main reason for the failed workpiece is ductile fracture. Thus the prediction of ductile fracture plays an important role for a forming process. Based on various hypotheses, many criteria for ductile fracture have been proposed empirically as well as theoretically $[16,17]$. According to cumulative damage theory, Cockcroft and Latham [18-20] proposed a damage computation model based on a critical value of the tensile strain energy per unit of volume, which has been extensively applied to predict fracture in various bulk forming processes such as extrusion, rolling, upsetting and so on. The equation is expressed as follows [18]:

$$
D=\int_{0}^{\bar{\varepsilon}_{f}} \frac{\sigma_{T}}{\bar{\sigma}} \mathrm{d} \bar{\varepsilon}
$$

where $\bar{\varepsilon}_{f}$ is the equivalent fracture strain, $\bar{\sigma}$ is equivalent stress, $\sigma_{T}$ is the maximal principal stress and $D$ is fracture threshold. This damage model is not based on a micromechanical model to estimate fracture, however, it simply recognizes the dependence of the critical at fracture upon the level of the largest principal stress. In eq. (1), the maximum damage value, $D_{\max }$, is named as DFC, which is the critical damage value of the workpiece. The maximum damage value is dependent on the material metallurgical properties such as grain morphology, grain size, and the inclusion content of non-metallic and so on. When the damage value of $\mathrm{D}$ reaches $D_{\max }$ during a forming process, ductile fracture will occur. Thus, by comparing $D$ with $D_{\max }$, the risk of material failure during forming processes can be assessed.

In order to identify the ductile fracture during a forming process, the value of $D$ was calculated by FE simulation. And eq. (1) has to be converted to an appropriate discrete expression which is convenient for $\mathrm{FE}$ code:

$$
D=\int_{0}^{\bar{\varepsilon} f} \frac{\sigma_{T}}{\bar{\sigma}} \frac{\mathrm{d} \bar{\varepsilon}}{\mathrm{d} t} \mathrm{~d} t=\int_{0}^{t_{f}} \frac{\sigma_{T}}{\bar{\sigma}} \overline{\bar{\varepsilon}} \mathrm{d} t \cong \sum_{0}^{t_{f}} \frac{\sigma_{T} \dot{\bar{\varepsilon}} \Delta t}{\bar{\sigma}}
$$

where $\dot{\bar{\varepsilon}}$ is the equivalent strain rate, $t_{f}$ is the total time corresponding to fracture failure, and $\Delta t$ is the variable time increment used in the FE analysis.

\section{Approach to determine Cockcroft-Latham type DFC}

From the true stress-strain curves of Ti-6Al-4V alloy, it can be found that Ti- $6 \mathrm{Al}-4 \mathrm{~V}$ alloy is a typical strain-softening alloy. It is well accepted that for strain-hardening alloys the fracture criteria can be determined by directly comparing the simulations with the destructive experiments in terms of the critical deformation level. However, for strain-softening alloy the DFC cannot be determined directly due to the fact that softening mechanisms such as dynamic recrystallization (DRX) and dynamic recovery (DRV) occur during the deformation processes, and it is difficult to find visible cracks on the surface of deformed sample and the stress-strain curves. Therefore, it is significant to find an indirect way to evaluate the ductile damage criteria for strain-softening alloy. In this research, an indirect research approach has been brought forward, which utilizes physical experiments, numerical simulations and mathematic computations to provide mutual support for determining the critical fracture time.

As a series of samples were compressed on a heat physical simulation machine under different seven deformation temperatures and four strain rates, the true stressstrain data were collected and then inputted in the FE software of DEFORM-2D. Through an integration method using Eq. (2) in the FE software of DEFORM-2D, the damage values at all strains during the simulation processes were obtained. According to cumulative damage theory, the damage value increases with increasing level of deformation, when it reaches the critical damage value, ductile fracture occurs. However, during the simulation procedure of the compression test in DEFORM-2D, the damage value will keep increasing until the last compression step, even if the damage value reaches the critical damage value. Therefore, it is necessary to analyze the damage accumulation process and identify the initial fracture time.

Kachanov [21-23] explained the "one-dimensional surface damage variable" by considering a damaged body and a representative volume element (RVE) at a point $M$ oriented by a plane defined by its normal $\vec{n}$ and its abscissa $x$ along the direction $\vec{n}$ (as shown in Figure 2) [21]. The value of the damage $D(M, \vec{n}, x)$ attached to the point $M$ in the direction $\vec{n}$ and the abscissa $x$ is:

$$
D(M, \vec{n}, x)=\frac{\delta S_{D x}}{\delta S}
$$




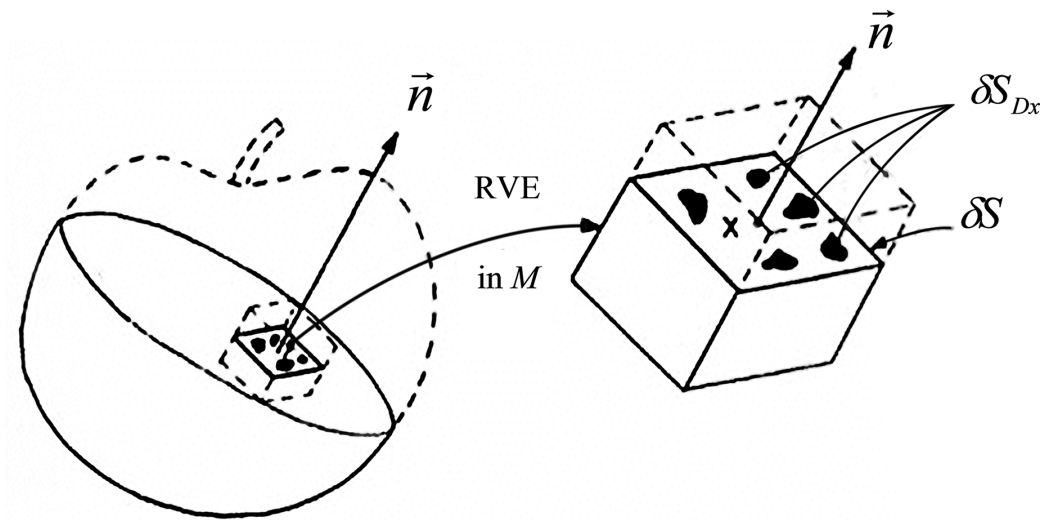

Figure 2: Damaged RVE in a damaged body.

where $S_{D x}$ is the area of intersection of all the flaws with the plane defined by the normal $\vec{n}$ and abscissa $x ; S$ is the total area at the intersection plane. Damage $D$ is bounded as $0 \leq D \leq D_{c}$, where $D_{c}$ is a critical damage value corresponding to the decohesion of atoms. $D=0$ represents the undamaged RVE material, and $D=D_{c}$ represents the rupture failure in the remaining resisting area. When the maximum damage value keeps increasing in a very small growth rate near to zero or equal to zero with increasing deformation strain, it means that fractures happens [24].

Based on Kachanov's one-dimensional surface damage theory, it can be known that the damage value increases with the increasing of time during a compressing simulation. Thus the maximum value seems to appear at the last simulation step which is not the initial fracture step. As the cumulation damage value contributes to identify the critical damage value, it is considerable to analyze the damage cumulating process. Thus, an innovative concept of the sensitive rate of Cockcroft-Latham damage $\left(R_{\text {step }}\right)$ in plastic deformation processes is developed as eq. (4) [12]:

$$
R_{\text {step }}=\frac{\Delta D}{D_{\text {acc }}}
$$

In which $R_{\text {step }}$ is equals to the ratio of the damage increment at one step $(\Delta D)$ to the accumulated value (Dacc). And it is supposed that if the maximum damage value keeps increasing in a very small growth rate near to zero, which means Rstep decreases gradually to zero or near zero, ductile fractures appears. The conception of such assumptions is of good consistency to Kachanov's explanation of damage mentioned above. And then the fracture time, that is, fracture strain or fracture height reduction can be identified and determined.

\section{Results and discussion}

\section{FE simulation of ductile damage and its analysis}

Rigid-plastic FE models were established in the DEFORM-2D platform to simulate the above corresponding compression tests. The basic FE model of compression test in DEFORM-2D is shown in Figure 3. Only one-half of a specimen is considered to simplify and reduce the running time during the simulation process because of the axisymmetry of compression. The specimen was modeled as a plastic object and the tools were modeled as rigid surfaces. The initial mesh
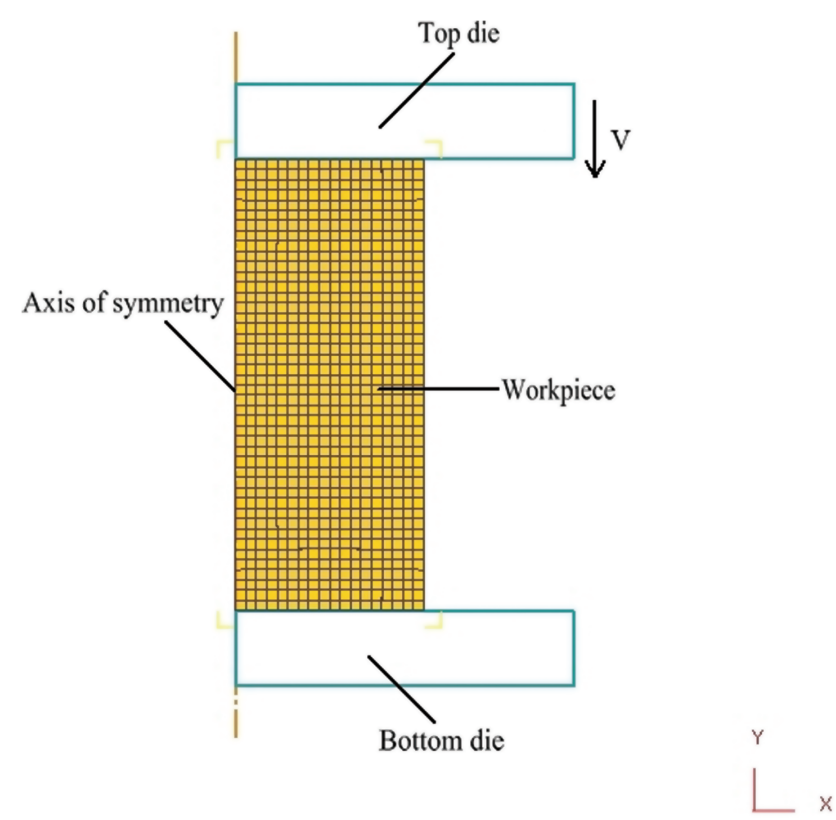

Figure 3: The basic FE model of compression testing in DEFORM-2D. 
of billet consists of 792 four-node elements. Due to both sides of the cylinder workpiece filled with machine oil mingled with graphite powder as lubrication in the hot compression test, the friction between the specimen and the tools is defined to be 0.1. For the simulation of plastic deformation process, the material model for billet can be defined by inputting the true stress-strain curve date (as Figure 1). Then the damage values during hot deformation process were calculated. Figure 4 shows the damage distribution of the height reduction $60 \%$ at the temperature of $1123 \mathrm{~K}$ and the strain rate of $0.1 \mathrm{~s}^{-1}$, it can be seen that the material in drum-shaped region is the most severely damaged, and the damage value decreasing sharply along the radius from outside to inside. From the simulation results of all the isothermal hot compression tests at the temperature of $1023 \mathrm{~K}$, $1073 \mathrm{~K}, 1123 \mathrm{~K}, 1173 \mathrm{~K}, 1223 \mathrm{~K}, 1273 \mathrm{~K}$ and $1323 \mathrm{~K}$, the strain rates of $0.01 \mathrm{~s}^{-1}, 0.1 \mathrm{~s}^{-1}, 1 \mathrm{~s}^{-1}$ and $10 \mathrm{~s}^{-1}$, it can be summarizes that the maximum damage value always appears in drum-shaped region corresponding to higher strain, stress and strain rate, while the minimal value appears in the center region. Therefore, the initiation of crack will occur at the edge of the sample.

Based on the simulation results, the evolution of damage value during the whole upsetting processes at seven temperatures and four strain rates were obtained and shown in Figure 5(a) (d). it is obvious that the damage value increases non-linearly with increasing strain from 0 to nearly 0.4 , and then it increases almost linearly until the end of deformation. Comparing these curves with one another, it is summarized that for a fixed true strain the maximum cumulated damage value decreases with increasing temperature at the strain rates of $0.01 \mathrm{~s}^{-1}, 0.1 \mathrm{~s}^{-1}$ and $1 \mathrm{~s}^{-1}$, while there is no obvious regular for the strain rate of $10 \mathrm{~s}^{-1}$. Besides, the maximum cumulated damage values under seven different temperatures and four different strain rates were illustrated in Figure 6, at the temperature of $1023 \mathrm{~K}, 1073 \mathrm{~K}, 1123 \mathrm{~K}$ and $1323 \mathrm{~K}$, as the strain rate increases, the maximum cumulated damage values slightly decrease firstly, then obviously increase, and lastly decrease again. When the temperatures are $1173 \mathrm{~K}, 1223 \mathrm{~K}$ and $1273 \mathrm{~K}$, the damage values increase with increasing strain rate in the beginning, and subsequently the damage values have a slight decrease. In further, it can be summarized that strain rate has a significant effect on the damage value, that is, the damage cumulating process is sensitive to strain rate.

\section{Evaluation of VDFC}

In order to evaluate the DFV, the initial fracture time and the damage value at fracture time are necessary to be ensured. By utilizing the damage values as shown in Figure 5 and the damage sensitive rate proposed as eq. (4), the sensitive rates $\left(R_{\text {step }}\right)$ of Cockcroft-Latham damage at seven temperatures and four strain rates were calculated and shown in Figure 7(a) (d). It can be seen that, for all these deformation conditions, $R_{\text {step }}$ decreases rapidly before true strain 0.1 , then decreases slowly and

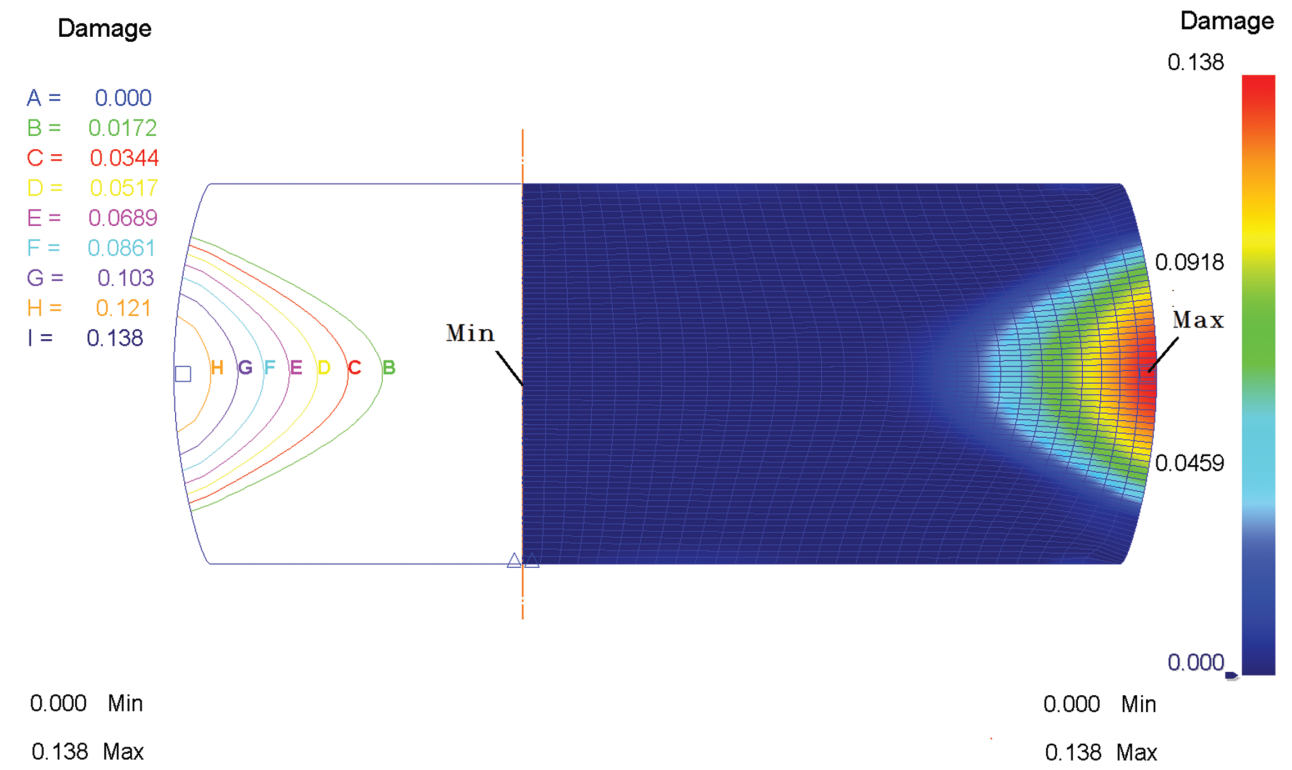

Figure 4: The damage distribution of the height reduction $60 \%$ at temperature of $1123 \mathrm{~K}$ and strain rate of $0.1 \mathrm{~s}^{-1}$. 


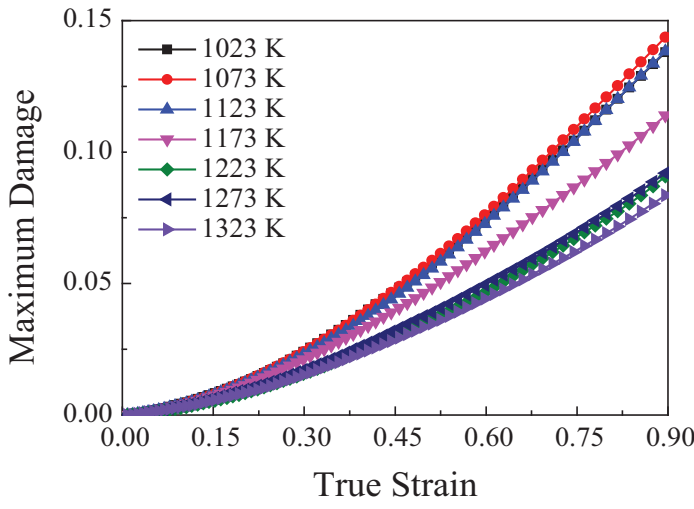

(a)

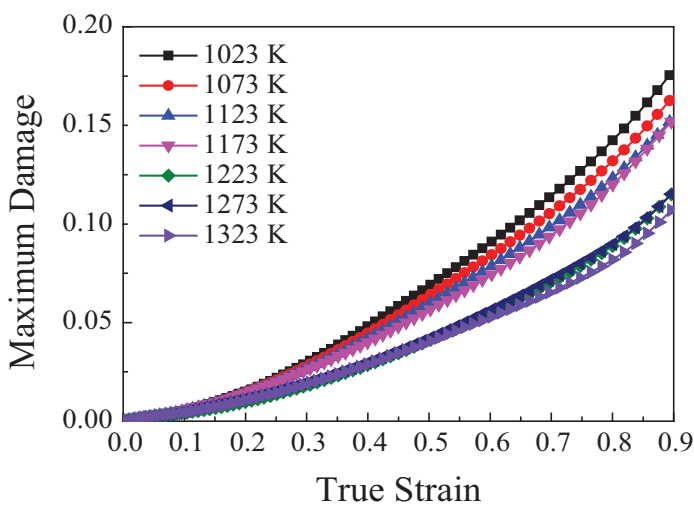

(c)

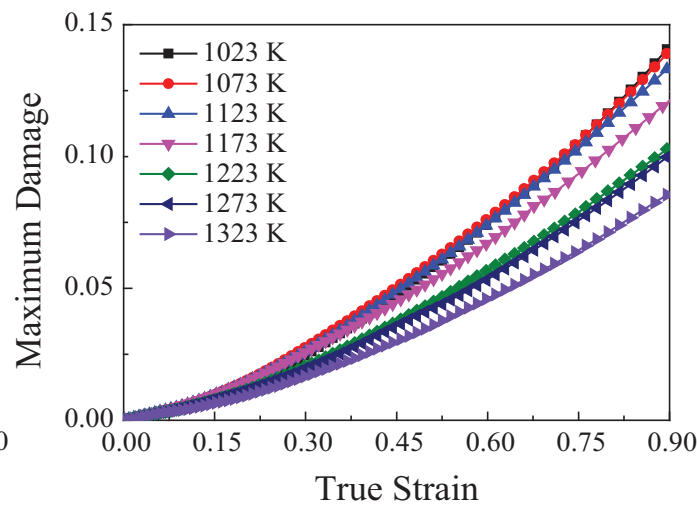

(b)

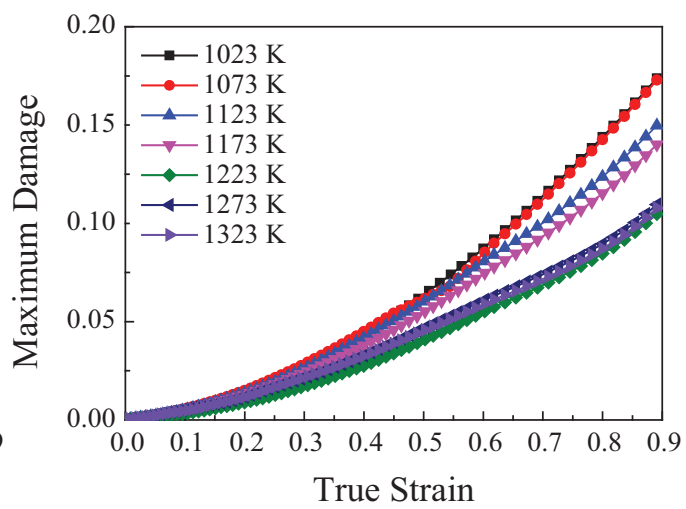

(d)

Figure 5: The maximum damage varying during compressing process at different temperatures and strain rates (a) $0.01 \mathrm{~s}^{-1}, 1023 \mathrm{~K} \sim 1323 \mathrm{~K}$; (b) $0.1 \mathrm{~s}^{-1}, 1023 \mathrm{~K} \sim 1323 \mathrm{~K}$ (c) $1 \mathrm{~s}^{-1}, 1023 \mathrm{~K} \sim 1323 \mathrm{~K}$; (d) $10 \mathrm{~s}^{-1}, 1023 \mathrm{~K} \sim 1323 \mathrm{~K}$.

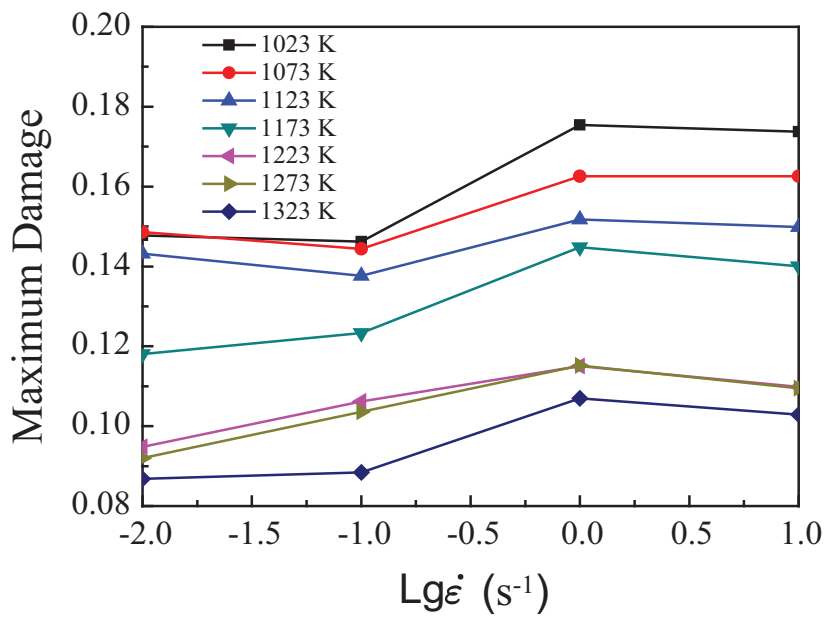

Figure 6: The relationships between maximum damage and strain rate at the end of compressing process under different temperatures.

even nearly zero. The point that $R_{\text {step }}$ equals to zero is assumed as the fracture time, and in this paper, the zero point has been added a tolerance $0 \sim 0.03$. That is, when $R_{\text {step }}$ decreases to 0.03 , it means that fracture occurs.
As shown in Figure 7, the point from $R_{\text {step }}-\varepsilon$ curves after true strain 0.5 were picked out and illustrated in amplifying scatter diagram so as to find the strain of initial fracture by determining the intersection of the curves and abscissa axis. In addition, the scatter diagrams without intersection with abscissa axis at the end of the simulation were fitted linearly, and the interception of the fitted line and abscissa axis was deeming as the initial fracture strain. As the initial fracture strain at each deforming condition obtained from Figure 7, the corresponding DFC under different deformation conditions can be identified by substituting the initial fracture strain in Figure 5. Figure 8 shows the response surface of $\mathrm{DFC}$, which is the maximum damage when the fracture occurs; it indicates the nature relationships between deforming conditions and DFC of Ti-6Al-4V alloy. Obviously, DFC of Ti-6Al-4V alloy under different temperatures and strain rates is not a constant, but a variable affected by the deformation conditions within a range of $0.07 \sim 0.15$. The DFC is sensitively dependent on deformation parameters involving strain rate and temperature. 

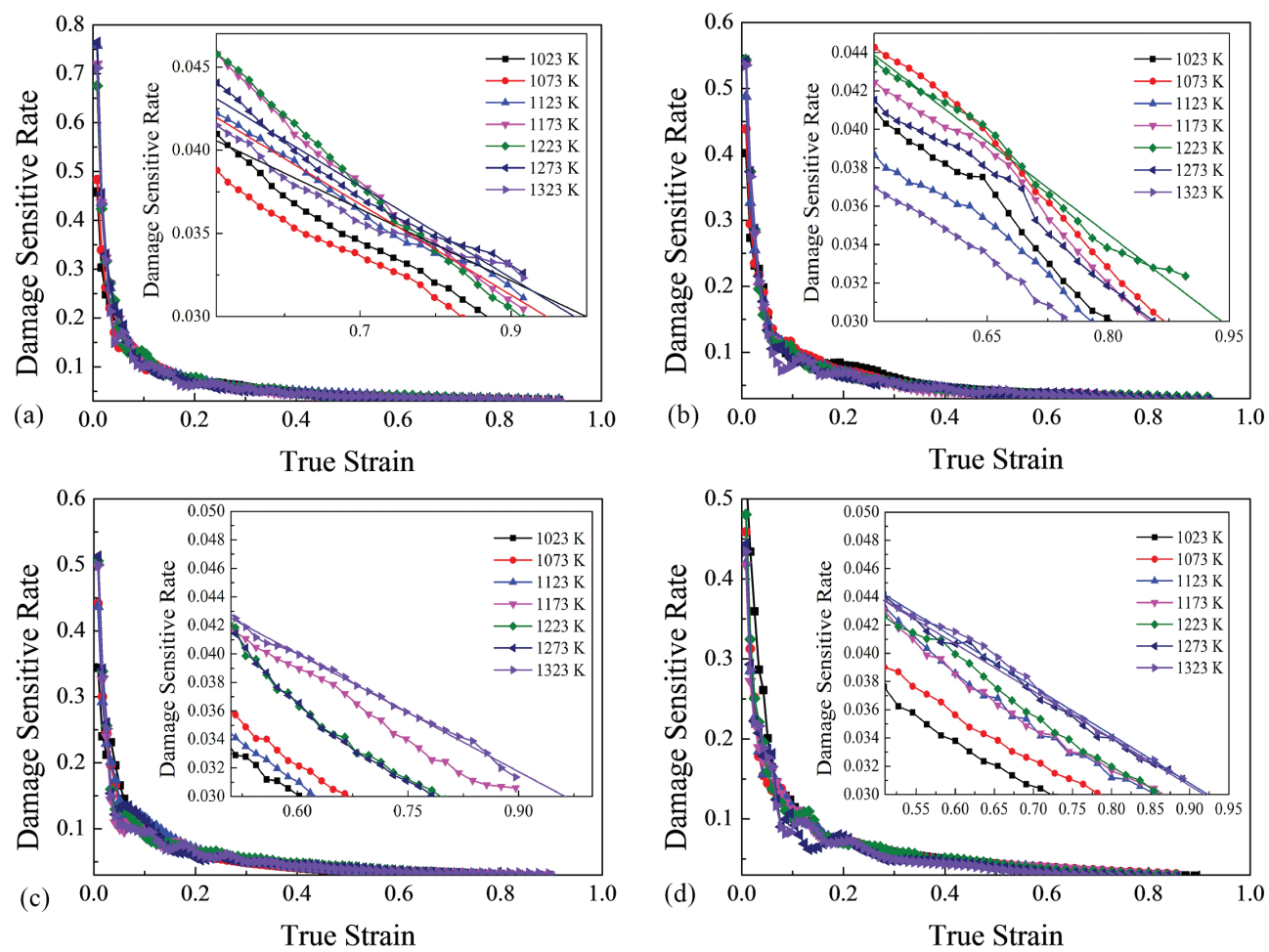

Figure 7: The variation of damage sensitive rate under different temperatures and strain rates (a) $0.01 \mathrm{~s}^{-1}$, (b) $0.1 \mathrm{~s}^{-1}$, (c) $1 \mathrm{~s}^{-1}$, (d) $10 \mathrm{~s}^{-1}$.

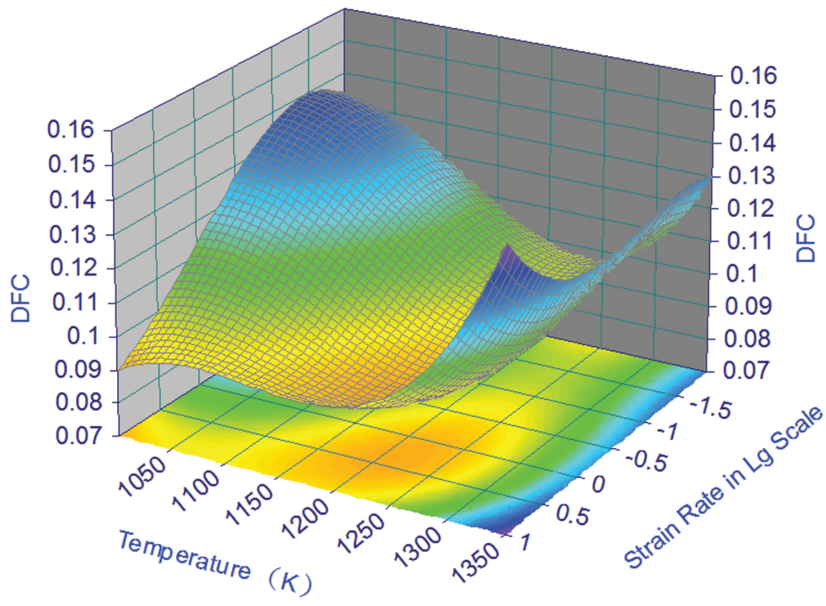

Figure 8: The response surface of the ductile fracture criteria of Ti$6 \mathrm{Al}-4 \mathrm{~V}$ alloy in temperature and strain rate.

Thus the DFC at different deforming conditions can be defined as VDFC and described as a function of strain rate and temperature, and the corresponding analytical formula was shown in Table 1.

It is found that there is a valley under the deformation conditions of 1200 1275 K \& 1 10 s ${ }^{-1}$ in Figure 8. On both sides of the valley, DFC values increase. It is notable that the workpiece will be fracture under these conditions due to lower DFC at the initial fracture. In addition to, the desired deformation domains (1023 1123 $\mathrm{K} \& 0.01 \sim 1 \mathrm{~s}^{-1}$ ) with lower fracture risk corresponding to higher DFC have been identified. Therefore, in bulk forming operations of Ti-6Al-4V alloy, fracture risk can be reduced by choosing suitable deformation parameters.

\section{Verification of VDFC by microstructure observations}

In order to verify the DFC, the typical microstructures of Ti-6V-4Al alloy lying in the lower DFC region, where exist a high risk of fracture under different deformation conditions, were observed by optical microscopy and shown in Figure $9(\mathrm{a}-\mathrm{c})$. Figure $9(\mathrm{a}-\mathrm{c})$ shows the microstructures. Figure 9(a) and (b) shows the microstructures under the condition of $1173 \mathrm{~K} \& 1 \mathrm{~s}^{-1}$ where the critical damage value is about 0.08 . Figure 9 (a) shows the microstructure in drum-shaped region of deformed specimen with a visible crack. Figure 9(b) shows the microstructure in the center region of deformed specimen, from which, it can be found that the crack also appears in the center region. By comparing Figure 9(a) with Figure 9(b), it can 
Table 1: An approximate analytical formula for the VDFC of Ti-6Al-4V alloy.

\begin{tabular}{|c|c|c|}
\hline VDFC & Exponents & Remarks \\
\hline$z=a+b x+c y+d x^{2}+e y^{2}+f x y+g x^{3}+h y^{3}+i x y^{2}+j x^{2} y$ & $\begin{array}{l}a=-3.0917795 \\
b=-0.10613801 \\
c=0.011646993 \\
d=-0.02625198 \\
e=-1.394332 e^{-5} \\
f=0.00011469866 \\
g=0.0045644533 \\
h=5.483145 e^{-9} \\
i=-9.6282066 e^{-9} \\
j=3.616165 e^{-5}\end{array}$ & Error $=0.0080175651$ \\
\hline
\end{tabular}

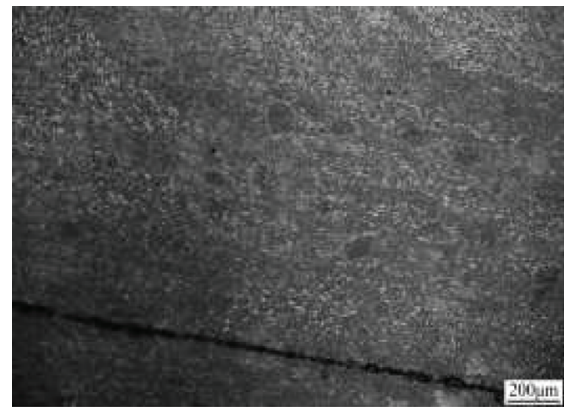

(a)

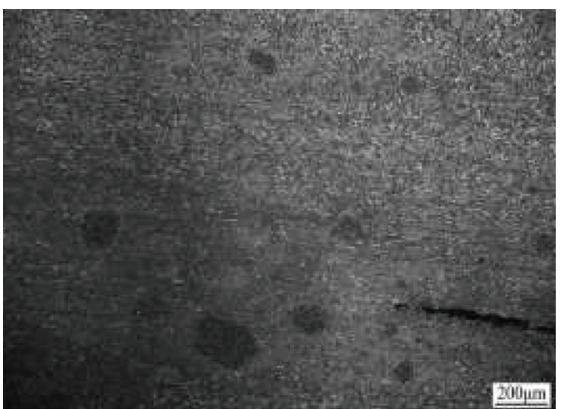

(b)

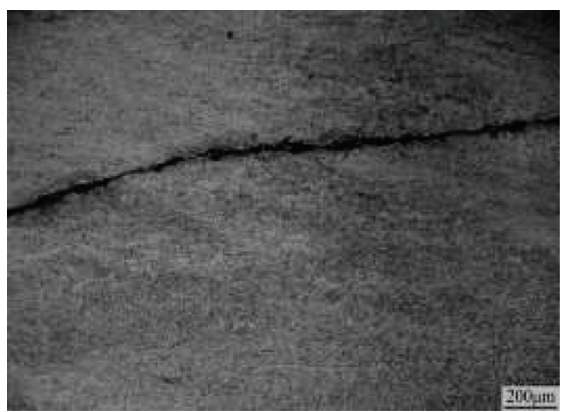

(c)

Figure 9: The microstructures of Ti-6Al-4V alloy under the deforming conditions (a) $1173 \mathrm{~K} \mathrm{\&} 1 \mathrm{~s}^{-1}$; drum-shaped region of deformed specimen, (b) $1173 \mathrm{~K} \mathrm{\&} 1 \mathrm{~s}^{-1}$; center region of deformed specimen, (c) $1273 \mathrm{~K} \mathrm{\&} 1 \mathrm{~s}^{-1}$; drum-shaped region of deformed specimen.

be summarized that the crack occurs in drum-shaped region firstly and then extends to the center, which is in good agreement with simulation results. Figure 9(c) shows the microstructure under the condition of $1273 \mathrm{~K}$ $\& 1 \mathrm{~s}^{-1}$, where the critical damage value is about 0.09 . From Figure 9(c), it can be found that a macro-crack appears in drum-shaped region, which shows a good agreement with the simulation. From these microstructures, it can be concluded that the VDFC is practical approach to predict the initial ductile fracture time of Ti-6Al-4V alloy and avoid the ductile fracture by optimizing processing parameters in the actual production.

\section{Conclusions}

Based on the criteria proposed by Cockcroft and Latham, the ductile damage cumulating process, fracture initiation sites and DFC diagram for Ti-6Al-4V alloy along with various deformation conditions were evaluated. The following conclusions were obtained:

(1) As for a typical strain-softening material of Ti-6Al-4V alloy, the maximum damage value appears in the drum-shaped region, while the minimal value in the center region. The fracture will firstly occur at the edge of the workpiece. 
(2) DFC of Ti-6Al-4V alloy in the temperature range of 1023 $\mathrm{K} \sim 1323 \mathrm{~K}$ and the strain rate range of $0.01 \sim 10 \mathrm{~s}^{-1}$ is not a constant but changes with a range of $0.07 \sim 0.15$. With the establishment of DFC diagram, the initial fracture time was identified. And then DFC was further defined as VDFC and characterized by a function of temperature and strain rate.

(3) Based on the VDFC diagram, the exact fracture moment during various deforming processes can be predicted effectively and conveniently. In addition, the deformation conditions with lower fracture risk of Ti-6Al-4V alloy were identified as $1023 \sim 1123 \mathrm{~K} \& 0.01$ $\sim 1 \mathrm{~s}^{-1}$, and the deformation conditions with higher fracture risk were identified as1200 1275 $\mathrm{K} \& 1 \sim 10 \mathrm{~s}^{-1}$.

Acknowledgements: The work was supported by Chongqing Foundation and Frontier Research Project (cstc2016jcyjA0335), Chongqing Basic Research and Frontier Exploration Project (cstc2018jcyjAX0459), Open Fund Project of State Key Laboratory of Materials Processing and Die \& Mould Technology (No.P2017-020), and Research Project of State Key Laboratory of Mechanical Transmission (No. SKLMT-ZZKT-2017M15).

\section{References}

[1] W. Peng, W. Zeng, Q. Wang and H. Yu, Mater. Des. , 51 (2013) 95-104.

[2] Y. Ning, M.W. Fu, H. Hou, Z. Yao and H. Guo, Mater. Sci. Eng. A, 528 (2011) 1812-1818.

[3] Y.B. Tan, J.L. Duan, L.H. Yang, W.C. Liu, J.W. Zhang and R.P. Liu, Mater. Sci. Eng. A, 609 (2014) 226-234.
[4] P.M. Souza, H. Beladi, R. Singh, B. Rolfe and P.D. Hodgson, Mater. Sci. Eng. A, 648 (2015) 265-273.

[5] D.J. Kim and B.M. Kim, Int. J. Adv. Manuf, Technol., 19 (2002) 336-342.

[6] D.C. Ko and B.M. Kim, J. Mater. Process. Tech., 102 (2000) 19-24.

[7] S.V.S.N. Murty, B.N. Rao and B.P. Kashyap, J. Mater. Process. Technol., 147 (2004) 94-101.

[8] X.Q. Zhang, Y.H. Peng and X.Y. Ruan, J. Mater. Process. Technol., 105 (2000) 253-257.

[9] B.P.P.A. Gouveia, J.M.C. Rodrigues and P.A.F. Martins, J. Mater. Process. Technol., 101 (2000) 52-63.

[10] H. Liu, Y. Yang, Z. Yu, Z. Sun and Y. Wang, J. Mater. Process. Technol., 209 (2009) 5443-5447.

[11] V. Tvergaard and A. Needleman, J. Mech. Phys. Solids. 40 (1992) 447-471.

[12] Y.F. Xia, G.C. Luo, D.S. Wu, G.Z. Quan and J. Zhou, Adv. Mechanical Eng., 2013,(2013-8-31) 2013 (2013) 520127-520127.

[13] G.Z. Quan, G.C. Luo, M. An, J.T. Liang and D.S. Wu, Sci. World J., 2014 (2014) 579328.

[14] G.Z. Quan, F.B. Wang, Y.Y. Liu, Y. Shi and J. Zhou, Trans. Nonferrous Metals Soc. China, 23 (2013) 749-755.

[15] Y.Q. Cheng, H. Zhang, Z.H. Chen and K.F. Xian, J. Mater. Process. Tech., 208 (2008) 29-34.

[16] S.E. Clift, P. Hartley, C.E.N. Sturgess and G.W. Rowe, Int. J. Mech. Sci., 32 (1990) 1-17.

[17] D. Dumont, A. Deschamps and Y. Brechet, Acta Mater, 52 (2004) 2529-2540.

[18] M.G. Cockcroft and D.J. Latham, J. Inst. Metals, 96 (1968) 33-39.

[19] M.J. Ward, B.C. Miller and K. Davey, J. Mater. Process. Technol., s 80-81 (1998) 206-212.

[20] W.F. Fan and J.H. Li, Mater. Sci. Eng. A, 499 (2009) 248-251

[21] D. Krajcinovic, Mech. Mater. , 8 (1989) 117-197.

[22] N. Bonora, D. Gentile and A. Pirondi, J. Strain Anal. Eng. Des., 39 (2004) 639-651.

[23] N. Bonora, D. Gentile, A. Pirondi and G. Newaz, Int. J. Plast., 21 (2005) 981-1007.

[24] Y.F. Xia, G.Z. Quan and J. Zhou, Trans. Nonferrous Met. Soc. China, 20 (2010) 580-583. 